\title{
Uterine balloon therapy for menorrhagia: A feasibility study of its use in the community setting
}

\author{
K F McAllister, MRCOG, DFFP, Consultant in Family Planning \& Reproductive Healthcare; A Bigrigg, MRCOG, MFFP, Clinical \\ Director, The Sandyford Initiative, 6 Sandyford Place, Glasgow G3 7NB, UK
}

Correspondence: Dr K F McAllister, Consultant in Family Planning \& Reproductive Healthcare, The Sandyford Initiative, 6 Sandyford Place, Glasgow G3 7NB, UK. Tel: +44 (0) 141211 8130. Fax: +44 (0) 141 211 8139. E-mail: Kay.McAllister@glacomen.scot.nhs.uk

(Accepted $15^{\text {th }}$ January 2002)

The Journal of Family Planning and Reproductive Health Care 2002: 28(3): 133-134

\begin{abstract}
Objectives. To determine whether uterine balloon therapy (UBT) for menorrhagia can be performed safely in the community setting, obviating the need for hospital admission or general anaesthesia.

Design. Prospective case studies of 20 women undergoing Thermachoice endometrial ablation for menorrhagia.

Setting. Glasgow Centre for Family Planning and Reproductive Health Care, Greater Glasgow Primary Care NHS Trust, Glasgow, UK.

Participants. Twenty women with menorrhagia unresponsive to medical therapy.

Main outcome measures. Pain levels experienced by women during the procedure, measured by visual analogue scores and analgesia requirements postoperatively.

Results. Pain scores were in the range 0.1-6.6 (median 1.1) for outpatient hysteroscopy, compared to 0.1-9.8 (median 4.0) for uterine balloon therapy. No procedure was abandoned due to pain.

Conclusion. UBT performed under local anaesthetic is tolerated well by patients. It is an effective treatment for menorrhagia, which is safe and easy to perform in the community setting.
\end{abstract}

\section{Key message points \\ - Menorrhagia can be investigated and treated in the community. \\ - UBT is safe and easy to perform in the community. \\ - UBT performed under local anaesthesia is acceptable to women.}

\section{Introduction}

Menorrhagia is one of the most common gynaecological conditions, defined as greater than $80 \mathrm{ml}$ blood loss per cycle. Almost half of all hysterectomies performed in the UK are for dysfunctional uterine bleeding (DUB). ${ }^{1}$ Women are often concerned about the possibility of cancer, therefore it is important to exclude pathology and provide rapid and effective relief of symptoms. Hysteroscopic surgery, with endometrial ablation by Nd:YAG (neodymium-yttrium aluminium-garnet) laser or resection, is effective compared to hysterectomy, resulting in reduced hospitalisation, morbidity and mortality with rapid recovery. ${ }^{2-5}$

UBT is a simple technique for endometrial ablation, with destruction of the endometrium into the myometrium. ${ }^{6} \mathrm{~A}$ large international multicentre study involving 296 completed cases provided no major complications and only $10(3 \%)$ minor postoperative complications such as fever, cystitis and endometritis. ${ }^{7}$
It is also a very effective treatment, with 1-year followup of 291 cases showing $62 \%$ having amenorrhoea/spotting or a significant reduction in menses and in a further $29 \%$ bleeding was controlled to an acceptable level. ${ }^{7}$ Since 1995 , more than 5000 procedures of Thermachoice balloon therapy have been performed worldwide with a success rate, defined as a reduction in bleeding to normal levels or less, of $86-91 \% .^{2,7-10}$

Our aim, by performing this procedure under local anaesthetic in a community setting, was to increase the acceptability of the procedure amongst women who may prefer treatment in a familiar environment, such as their general practitioner (GP) surgery or family planning clinic.

\section{Methods \\ Participants}

Women attending our community gynaecology clinic with menorrhagia unresponsive to medical therapy were selected for UBT. These included women who had tried oral progestogens, tranexamic acid, mefenamic acid, combined oral contraceptives (COCs) and Mirena intrauterine systems. The clinic is situated within a large family planning clinic. All the participants had undergone a prior local anaesthetic diagnostic hysteroscopy and endometrial sampling using the Pipelle sampler. Those women with abnormal pathology such as complex glandular hyperplasia or adenocarcinoma were excluded. A transvaginal ultrasound scan was performed and those with any fibroids greater than $4 \mathrm{~cm}$ were excluded, as were those with an endometrial cavity greater than $10 \mathrm{~cm}$. All women had a normal cervical smear within the previous 3 years. All ablation techniques can result in serious complications with future pregnancies therefore those women wishing to retain their fertility were excluded from the study. All had either female sterilisation or vasectomy. Any patients with a known or suspected latex allergy were not offered this treatment. Both hysteroscopy and UBT were performed in the same setting and by either one of two designated operators. It was made clear to the women that the procedure could be halted at any stage if they found it too uncomfortable or painful.

\section{Equipment and operative technique}

We used the Thermachoice (Gynecare Limited) system for endometrial ablation. It consists of a $16-\mathrm{cm}$ long catheter, which is $4.5 \mathrm{~mm}$ in diameter. The heating element is within a latex balloon at the distal end. The Thermachoice system has a central control unit, which monitors the intra-balloon pressure and temperature. The system deactivates 
automatically if the pressure is outside the safety levels of 45 to $200 \mathrm{mmHg}$. The procedure time and intra-balloon temperature and pressure are clearly displayed on the control unit.

All women were prescribed danazol $400 \mathrm{mg}$ daily for endometrial preparation, ranging from 1 to 3 months prior to the procedure. They were given a $100 \mathrm{mg}$ diclofenac sodium suppository to self-administer 1 hour prior to attending. All procedures were performed in the modified lithotomy position. No premedication was administered. A paracervical block was achieved using $10 \mathrm{ml} 1 \%$ bupivacaine. The cervix was grasped with a tenaculum and the balloon then inserted transcervically to the fundus. Dilation of the cervix to No. 5 Hagar was performed in three patients as we were unable to insert the balloon easily. Working strictly to the established guidelines, the balloon was inflated with $5 \%$ dextrose until the intra-balloon pressure stabilised between 160 and $180 \mathrm{mmHg}$. The heating cycle was then initiated and the 8-minute therapy cycle commenced when the intra-balloon temperature reached $87^{\circ} \mathrm{C}$. This is known to result in a $0.4-0.6 \mathrm{~cm}$ depth of tissue coagulation. ${ }^{6}$ All procedures were completed satisfactorily. A nurse was present beside the women throughout the procedures, talking to her and helping her relax. Following the procedure, the women were taken to a separate consulting room where they were allowed to recover prior to discharge. They were all given diclofenac sodium $50 \mathrm{mg}$ tablets to use at home if required.

\section{Assessment}

Visual analogue scores were used immediately following the procedures to assess pain scores experienced by the women. These were then compared with similar visual analogue scores obtained immediately following the local anaesthetic hysteroscopies. All women were then contacted by telephone the following morning to determine their analgesia requirements at home. The time spent in the clinic following the procedure was also noted.

\section{Results}

One patient was nulliparous. The others had a median parity of 2 (range $0-5$ ). The mean age was 40 years (range 23-49 years). Pain levels were assessed on a scale of 0 to 10 with 10 being the worst pain imaginable and 0 no pain. Pain experienced during hysteroscopy was in the range 0.1-6.6 with a median of 1.1 , compared to $0.1-9.8$ for Thermachoice with a median of 4.0. The procedures were tolerated well by the women. Almost all left our clinic within an hour of completion of the procedure (median 40 minutes, range 30-65 minutes). Once at home, all women required further analgesia. Eighteen had $50 \mathrm{mg}$ diclofenac sodium, one taking this only once, five taking a second dose and 12 requiring three doses over the 24 -hour period. The remaining two patients did not take the prescribed medication but did take an alternative (ibuprofen and Tylex).

No complications occurred during the procedures or postoperatively. Once at home no patient required further medical help, either from their GP or local hospital.

\section{Discussion}

The effectiveness of UBT to control menorrhagia secondary to DUB is not in doubt, and has been demonstrated elsewhere. ${ }^{8,10}$ It is highly effective, with success rates for the treatment of menorrhagia secondary to dysfunctional uterine bleeding in the range $80-90 \%$. These results are comparable with endometrial resection, rollerball and microwave endometrial ablation. However, unlike balloon therapy, these techniques necessitate the use of general anaesthesia.

All procedures were undertaken by a gynaecologist with the presence of one nurse to attend to the patient. The technique involved no special adaptations to the clinic. It may therefore be possible to offer this service at the GP level.

UBT can lead to a significant release of prostaglandins from the uterus. It is therefore important that patients be given non-steroidal anti-inflammatory medication prior to and after the procedure. It was reassuring for us that no patients felt the need to seek further medical assistance once at home. This is especially so with the service that we have, as there is no 24-hour cover as in the hospital service.

We do, however, acknowledge that good patient selection is required prior to undertaking the procedure. The initial hysteroscopy is certainly an aid in determining patient suitability. Any patient who is a candidate for rollerball ablation is a candidate for UTB therapy.

The potential cost savings of this treatment should not be overlooked. ${ }^{11,12}$ This not only includes medical, nursing and ancillary staff in hospitals, but the time off work for treatment and recovery of the woman. The current pressures on theatre use are also a significant factor. The actual time required to perform the procedure is 8 minutes. In our experience, approximately 45 minutes should be allocated for each patient.

We acknowledge that the number of patients treated under this protocol is small and a larger number would be required to determine the precise percentage of women in whom the pain is extremely severe. We did not have to abandon the procedure in any of these women. Larger participant numbers would also allow us to clearly demonstrate patient satisfaction levels.

\section{Conclusions}

The Thermachoice system for UBT is a simple, safe and easy procedure to perform. We found no indication why it cannot be offered in the primary care setting under local anaesthesia as an outpatient procedure involving minimum staff input. It was an acceptable form of treatment for DUB in our patients.

\section{Acknowledgements}

Thanks are due to all the women who participated in the study knowing that it was a new technique.

\section{Statements on funding and competing interests}

Funding. Gynecare Limited supplied Thermachoice devices free of charge. Gynecare Limited had no input into the study design or the evaluation of the results

Competing interests. None declared.

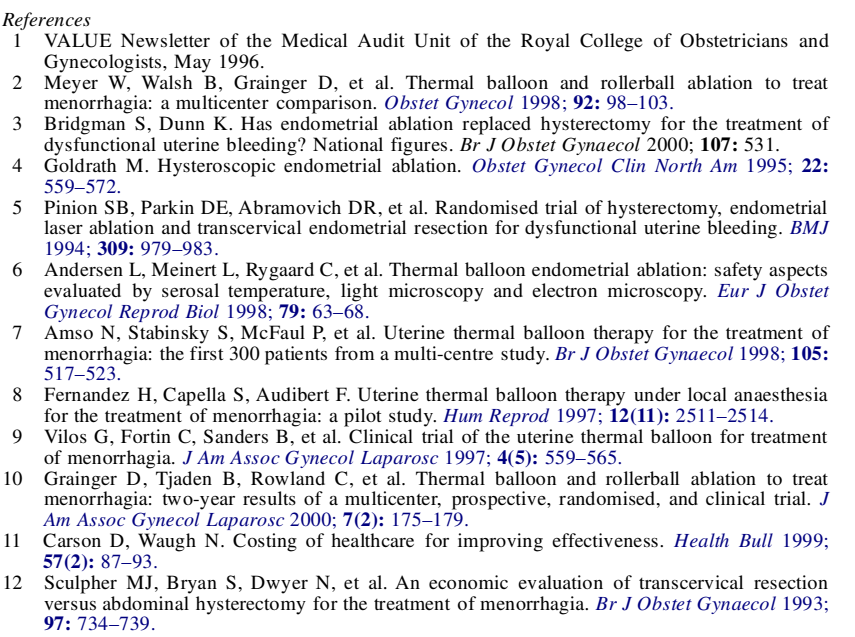

Obstet Gy replal $1998,92: 98-103$. dysfunctional uterine bleeding? National figures. Br J Obstet Gynaecol 2000; 107: 531. Goldrath

(a) endometrial

Andsen L, Meinert L, Rygaard C, et al. Thermal balloon endometrial ablation: safety aspects valuated by serosal temperature, lig menorrhagia. J Am Assoc Gynecol Laparosc 1997; 4(5): 559-565. enorrhagia: two-year results of a multicenter, prospective, randomised, and clinical trial. $J$ Sculpher MJ, Bryan S, Dwyer N, et al. An economic evaluation of transcervical resection 97: 734-739. 